\title{
DESIGN OF H-INFINITY TRACKING CONTROLLER FOR APPLICATION IN AUTONOMOUS STEERING OF MOBILE MACHINES
}

\author{
Alexander Mitov, Jordan Kralev, Tsonyo Slavov, Ilcho Angelov \\ Technical University of Sofia, Bulgaria \\ a_mitov@tu-sofia.bg,jkralev@tu-sofia.bg
}

\begin{abstract}
The purpose of the designed $H_{\infty}$ controller is to drive effectively the hydraulic system and to make it follow the desired trajectory, which is commanded by a joystick, steering wheel or geographic based data. To design the controller we used the experimentally identified SIMO linear mathematical model. The identification procedure is based on random excitation signals and prediction error methods for regressive discrete-time equations. The input of the mathematical model is the voltage applied to the spool driver EH module (PVE) and the outputs of the model are the spool position of the proportional valve in the steering unit, flow rate and the cylinder piston position. These output variables carry important information about the state of the system and can be used to increase performance in respect to the case, when using only the cylinder piston position or pressure signals. We have already experimented with various control strategies for the identified mathematical model. Experimental studies are performed on a laboratory test rig for electrohydraulic steering systems based on the 32-bit microcontroller and taking into account the technical specifications of mobile machine manufacturers and standards. In addition to the requirements of the safety standards, new advantages are achieved in terms of precise tracking control and providing a variable steering ratio between the steering wheel and the steering cylinder supported by the designed $H_{\infty}$ controller.
\end{abstract}

Keywords: H-infinity controller, tracking, autonomous steering, mobile machines.

\section{Introduction}

Electrohydraulic steering unit (EHSU) for low-speed mobile machines meets higher functional, safety, reliability and relatively low cost. For example, we can point to EHPS - Electro Hydraulic Power Steering. These types of systems allow for two main modes of operation for path tracking depending on the formation of the control action. One mode is mechanical, in which the control action form is via the steering wheel, and the other is digital via a joystick panel, programmatically or GPS. The EHSU is mainly composed of a load-sensing valve and a proportional spool valve is integrated in single assembly unit and works synchronously. Position sensors of the proportional valve and steering wheel support feedback control to achieve variable transmission ratios [1]. Therefore, the steering wheel feedback reaction torque can be altered as a function of current machine state. In addition, unexpected angular rotations of the steering wheel can be removed.

Steering control systems are significant, because loading torques acting upon the steering axle may disturb steering performance. Also, the mathematical model cannot account for all physical details, because it would become impractically complex. The most appropriate means for development of a single input multiple outputs (SIMO) linear plant model, which does not require detailed a priory information, is the so called "black box" system identification [2]. The input to the mathematical model is the voltage applied to the spool driver EH module (PVE) and the outputs of the model are the spool position of the proportional valve in the steering unit, flow rate and the cylinder piston position. The outputs carry information about the hydraulic steering system in different frequency ranges. The spool position signal is located at the highest frequencies, since the relatively low mass of the spool and its direct access to the pressure is supplied by the fixed displacement pump. The flow signals are slower as dynamics compared to the spool position signal, since an amount of time is required to accelerate the working fluid through the geometrical features of the hydraulic system. The flow rate signal is proportional to the piston velocity. The slowest dynamics has the piston position signal that is integral of piston velocity.

We have already experimented with various control strategies for the identified mathematical model - cascade control based on PID and linear quadratic Gaussian (LQG) controller. The PID control is appropriate for manual tuning, but it cannot reach the optimal performance of the closedloop tracking system. The achieved steady-state error and settling time are limited with the PID, of course, more effort in its tuning can alleviate this. On the other hand, the LQG controller gives optimal closed-loop performance for a particular choice of $\mathrm{Q}$ and $\mathrm{R}$ matrices. However, this optimal performance is guaranteed only in statistical sense for the system response on the average, since $\mathrm{H}_{2}$ 
norm of the closed-loop is minimized [3]. However, such optimization may not guarantee that some considerable deviations from tracking performance can happen, even with very small probability. Such possibility may be acceptable for some of applications of hydraulic servo systems [5], but it is not so appropriate for the hydraulic steering system. Therefore, in this case we try to minimize the $H_{\infty}$ norm of the closed-loop system. The $H_{\infty}$ norm of a system is defined as

$$
G_{\infty}=\sup _{\omega} \frac{y(j \omega)_{2}}{u(j \omega)_{2}}=\sup _{\omega} \bar{\sigma}(G(j \omega)),
$$

which represents the worst case gain of the system $G$ over the frequency range and it equals the supremum of the maximal singular value of the system $G$ over the frequency range. Since reliability and safety are of primary importance in hydraulic machine design, the automatic steering control has to satisfy increased robustness requirements [6]. The purpose of the $H_{\infty}$ controller $K$ is to minimize the $H_{\infty}$ norm of the weighted closed-loop system or mathematically stated

$$
\inf _{K} \sup _{\omega} \bar{\sigma}\left(F_{L}(G(j \omega), K(j \omega)),\right.
$$

where $F_{L}$ - signifies the lower fractional transformation commonly used to represent the calculation of closed-loop models for multivariable systems.

The system model $G$ has one input and three outputs as we commented above. The controller $K$ has three inputs (spool position, flow rate signal, piston position error) and one output. As we can see, the $H_{\infty}$ controller design problem is a min-max optimization problem, which does not have analytic solution[7]. So, the design procedure is iterative based on a bisection algorithm, which minimized the value of the upper bound $\gamma$ such that

$$
\sup _{\omega} \bar{\sigma}\left(F_{L}(G(j \omega), K(j \omega))<\gamma .\right.
$$

The validity of the above equation can be checked for some value of $\gamma$ related to the existence of solution $X_{\infty}$ to controllability and $Y_{\infty}$ to observability Riccati equations. Then the controller $K(j \omega)$ is constructed as a state feedback with gain $K_{\infty}=X_{\infty} Y_{\infty}$ and the state is estimated by a so-called worstcase observer [8].

The main goal of this article is to present the design and implementation of an advanced version of an embedded $H_{\infty}$ path tracking controller for application in autonomous steering of mobile machines. The developed by the authors first version of the embedded control system with this type of controller contains feedbacks in the pressure drop in the two chambers of the steering cylinder and the position of its piston. Due to the low sensitivity of the $H_{\infty}$ controller to the pressure output system channel, in this work a controller with additional feedback inputs at the spool position of the proportional valve (integrated in the steering unit) and flow rate supplied to the unit was developed.

\section{Laboratory test rig layout}

- Electro-hydraulic steering.

The authors have designed and implemented a laboratory test rig for studying EHSU type OSPEC200 LSRM, according to the technical data sheet from the manufacturer. The designed hydraulic circuit diagram and detailed description of the test rig system is presented in [9].

- Embedded control system.

The architecture of the extended computational system for real-time implementation of the advanced controllers is presented in Figure 1. The industrial controller for mobile applications of MC012-022 is responsible for data acquisition and physical signal manipulation. In our case, the measured signals are the proportional spool position, flow rate to EHSU and the cylinder position. The output signal from the controller is PWM voltage applied to the PVE electro-hydraulic transducer. The second component of the extended computation system is the CAN communication channel between the MC012-022 microcontroller and the Simulink ${ }^{\circledR}$ real-time simulation model, which is deployed on the conventional workstation. The idea is to support a real-time communication, which extends the computational capabilities of the microcontroller with those of the dedicated workstation. Such 
architecture requires reliable data transmission of data rate, which is considerably higher than the sampling interval of the control system. So, within that period of $10 \mathrm{~ms}$ the message is transmitted to the Simulink ${ }^{\circledR}$ model running on the workstation for a time $\tau_{1}$, processed there for a $\tau_{2}$ and a response is returned back to the microcontroller for a time $\tau_{3}$. The real-time critical element in so described communication channel is the workstation processing. However, if we assume that the workstation is fast enough, then we have guaranteed the response time $\tau_{1}+\tau_{2}+\tau_{3}<0.01$. This makes the system to function in real-time.

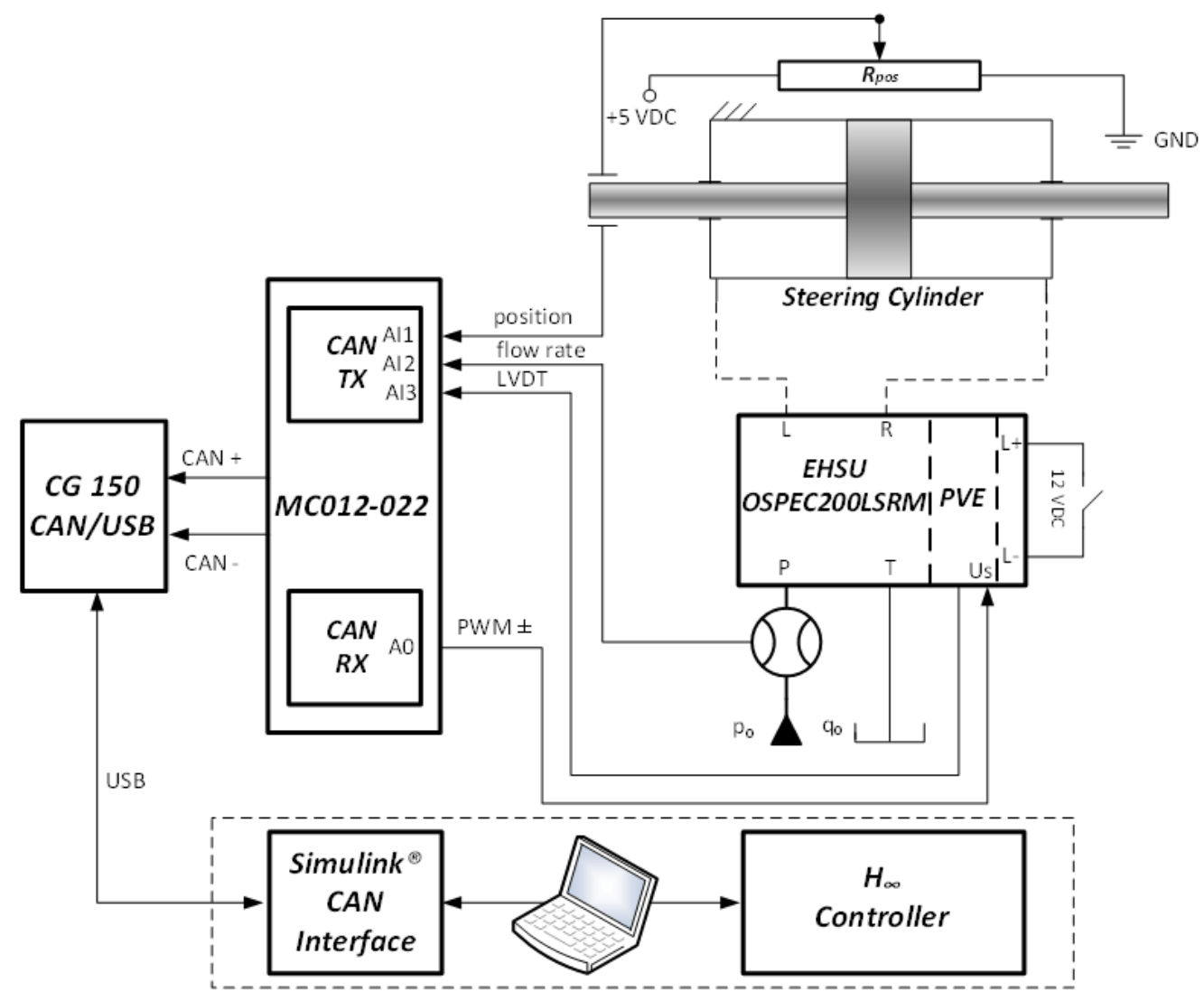

Fig. 1. Extended computational system for real-time implementation of advanced controllers

The Simulink ${ }^{\circledR}$ model used for controller implementation according to the above diagram is configured to run in continuous or discrete-time simulation mode with fixed step size equal to $10 \mathrm{~ms}$ to correctly measure the time. We have developed a specialized communication block, which interfaces with the CG150 USB/CAN communication cable converter. The communication block uses MATLAB $^{\circledR}$ Vehicle Network Toolbox, which includes components for CAN communication adapted for various vendors. The developed specialized communication block receives CAN messages in a blocking mode. Hence, the execution of the simulation model is blocked until the message synchronized with the $10 \mathrm{~ms}$ time frame arrives. Then the model blocks are executed for one step, and when the execution of the communication block is in order, it blocks simulation again till arrival of the consequent CAN message from the MC012-022 microcontroller. Since the computational capabilities of the workstation can be practically extended without limits, the proposed architecture allows for implementation of more complicated and computationally demanding controllers.

\section{Design of H-infinity controller}

- System identification model.

We have estimated a discrete-time state space model with the help of random binary input sequence (RBS) identification experiment during open-loop operation of the electrohydraulic steering system [10]. The experiment was conveyed with a sample interval of $25 \mathrm{~ms}$, and the estimated model is then converted to a continuous time model for the controller design with the help of bilinear Tustin transform. 
The resultant state-space model used for design of $H_{\infty}$ controller has the form

$$
\begin{aligned}
& \mid \begin{array}{l}
\dot{x} \\
y=A x+B u \\
y=C x+D u
\end{array}, A=\left(\begin{array}{ccc}
-171.9 & 16.46 & 557.1 \\
-1.764 & 0.02159 & 8.835 \\
-33.02 & 3.105 & 103.3
\end{array}\right), C=\left(\begin{array}{ccc}
-31 & 0.67 & -6.58 \\
-0.584 & 0.007 & -0.25 \\
-0.059 & -2.019 & 0.125
\end{array}\right), \\
& B=\left(\begin{array}{llll}
0.02716 & -0.006449 & -0.001482
\end{array}\right)^{T}, D=\left(\begin{array}{lll}
0.000117 & 1.4 \times 10^{-5} & 2.1 \times 10^{-6}
\end{array}\right)^{T} .
\end{aligned}
$$

The identified model has three outputs and a single input. The input is the voltage level applied as a PWM signal to the PVE transducer, the first output is the LVDT position, the second output is the flow rate through EHSU and the last output is the cylinder piston position. The model is stable, but non-minimal phase on all channels, which limits the achievable dynamical response of the closed-loop system. This can be seen from the zero-pole representation of the above model

$$
\begin{aligned}
& W_{\text {lvdt }}(s)=\frac{y_{1}(s)}{u(s)}=\frac{0.000117(s-6900)(s-149.5)(s+0.2172)}{(s+57.44)(s+10.92)(s+0.1992)}, \\
& W_{\text {flow }}(s)=\frac{y_{2}(s)}{u(s)}=\frac{1.4 \times 10^{-5}(s-829.7)(s-209)(s+0.2221)}{(s+57.44)(s+10.92)(s+0.1992)}, \\
& W_{c y l}(s)=\frac{y_{3}(s)}{u(s)}=\frac{2.1 \times 10^{-6}(s+28.63)\left(s^{2}-191.5 s+1.41 \times 10^{5}\right)}{(s+57.44)(s+10.92)(s+0.1992)} .
\end{aligned}
$$

- $H_{\infty}$ synthesis.

In order to synthesize the $H_{\infty}$ controller we first define an extended open-loop system including weighting functions, with the help of which we can specify the performance requirements of the closed-loop. The extended plant has the form

$$
\dot{x}_{e x t}=A_{e x t} x_{e x t}+B_{e x t}\left(\begin{array}{l}
r \\
u
\end{array}\right),\left(\begin{array}{c}
z_{u} \\
z_{y} \\
e
\end{array}\right)=C_{e x t} x_{e x t}+D_{e x t}\left(\begin{array}{l}
r \\
u
\end{array}\right),
$$

where $x_{e x t}=\left[x_{1}, x_{2}, x_{3}, x_{u}, x_{e}\right]$. We selected the following weighting filters based on observation of open-loop and closed-loop performance

$$
W_{u}=2 \times 10^{-5} \frac{s+1}{0.1 s+1}, \quad W_{e}=\operatorname{diag}\left(\begin{array}{lll}
0.1 & 1 & 20
\end{array} \frac{0.1 s+1}{3 s+1}\right),
$$

where $W_{u}$ and $W_{e}$ - used to weight the control action and the control system error respectively.

A central limiting factor is the dead-band of the main spool into the EHSU. It is introduced to prevent from random steering drifts in case of exploitation wearing and too small operator commands. The dead-band limits the performance, because if the loop gain is too low, the reference value will not be reached. And, if the loop gain is too high, then high frequency oscillations are excited upon the steering cylinder piston. This is undesired effect, because such vibrations will be transmitted through the whole steering mechanism up to the wheels. One way to control the amplitude and the frequency of the oscillations is to increase the input weighting filter gain $W_{u}$ at high frequencies, however, this could limit the bandwidth of the closed-loop system and increase the response time. However, the identified model allows us to increase the gain on the flow rate, which turns to have very positive effect upon attenuating the oscillations. The purpose of the $H_{\infty}$ controller is to minimize the $H_{\infty}$ norm of the closed-loop, which is determined with the maximal singular value of the loop [7]. This problem becomes of the min-max type, so it does not have general solutions, so we look for suboptimal solutions for a fixed upper bound of the $H_{\infty}$ norm. In the present research we use a method for its decomposition to two Riccati equations - corresponding to observability and controllability of the extended plant and its dual. This allows for check whether a controller exists for a particular value of the $H_{\infty}$ norm. Starting with some high value like 100 we have achieved with bisection the value under 2. This means that the requirements of the closed-loop performance are satisfied well enough. Fig. 2 presents the step response of the closed-loop with the $H_{\infty}$ controller and Fig. 3 shows the output sensitivity of the cylinder piston channel compared with the respective weighting function. 


\section{Experimental study of $\mathbf{H}$-infinity controller}

The Simulink ${ }^{\circledR}$ diagram used to perform experiments with the $H_{\infty}$ controller is presented in Figure 4 [4]. The central block is MATLAB ${ }^{\circledR}$ function block, which supports the communication on the CAN channel between MC012-022 and the workstation. Also, this block guarantees that the model will run in a blocking mode. Since the synthesized $H_{\infty}$ controller is continuous, it is discretized with the sample time $10 \mathrm{~ms}$.

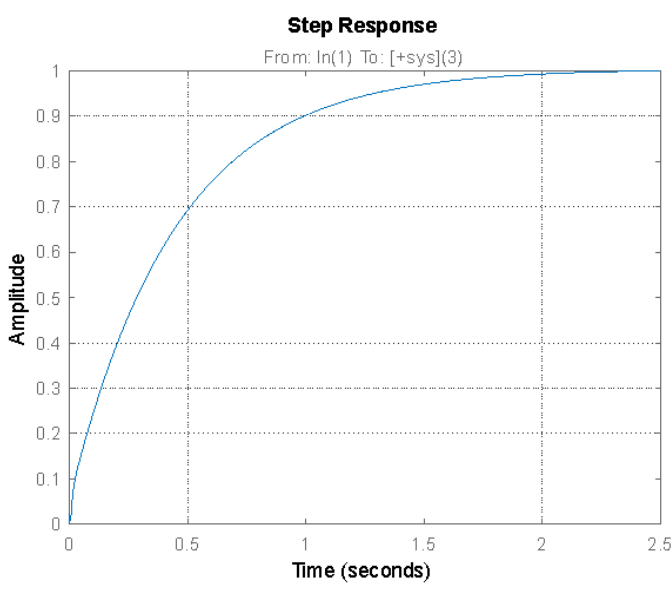

Fig. 2. Step response of closed-loop system

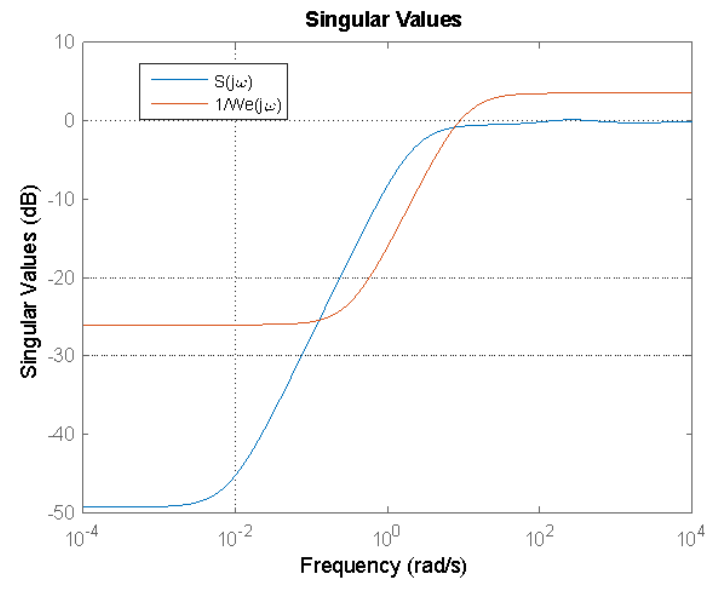

Fig. 3. Singular values of closed-loop system

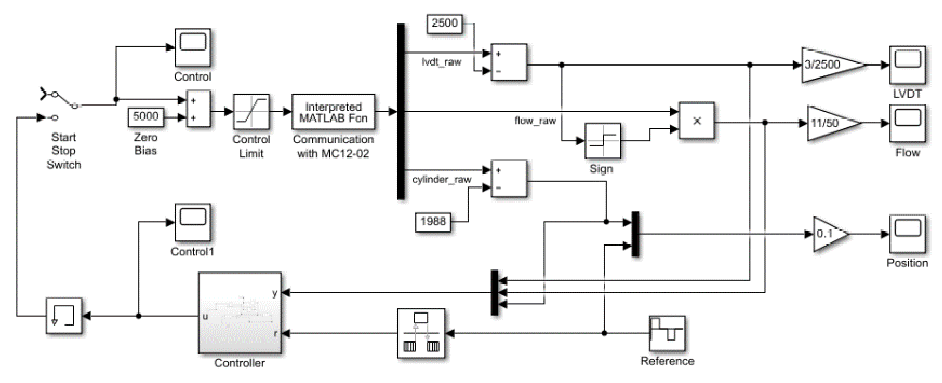

Fig. 4. Simulink ${ }^{\circledR}$ diagram for implementation of the $H_{\infty}$ controller in real-time

In Fig. 5, the comparison between the reference and the piston position measured from the advanced version of $H_{\infty}$ controller is recorded. The controller provides the closed-loop transient response with zero steady-state error and without overshot and oscillations. The good performance of the cylinder position comes on the cost of the high frequency control signal, which can be observed in Fig. 6. However, these oscillations are within acceptable limits. The measured LVDT signal is presented in Fig. 7, and its shows that spool movementfollows the control signal. Fig. 8 shows the measured flow rate during the step reference tracking. The graphical result shows that the maximum flow rate the servo system reaches is $81 \cdot \mathrm{min}^{-1}$ in comparison with the pump flow rate -approximately $301 \cdot \mathrm{min}^{-1}$.This fact proves the energy efficiency of the system and creates a prerequisite for energy analysis.

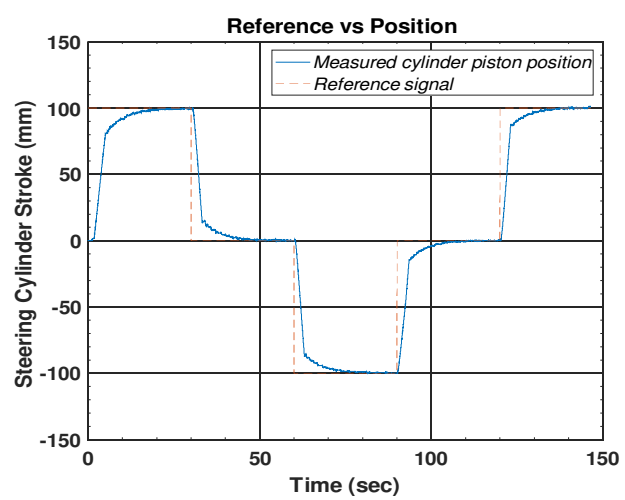

Fig. 5. Measured step reference tracking

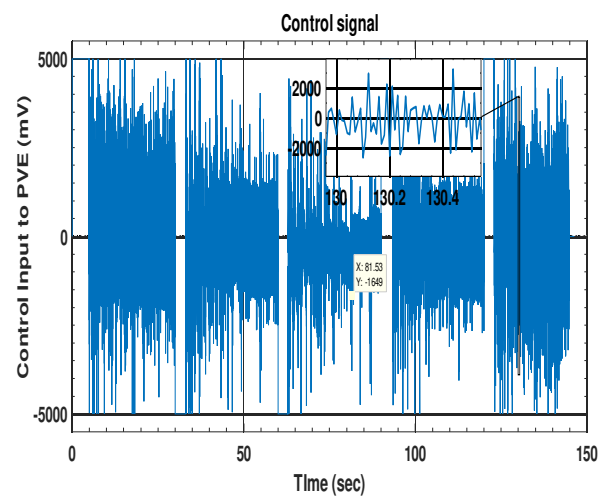

Fig. 6. Measured control action 


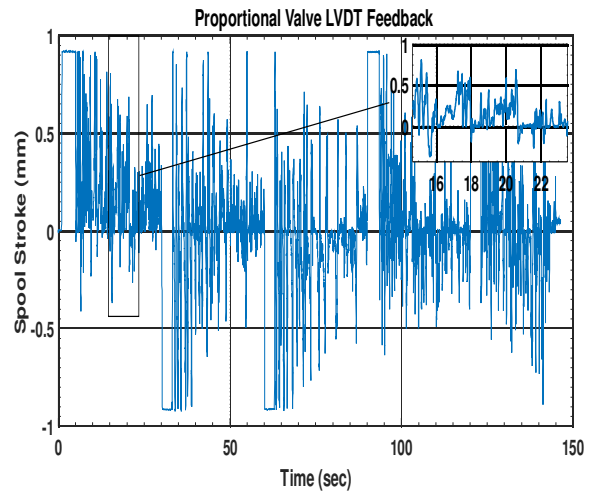

Fig. 7. Measured LVDT feedback signal

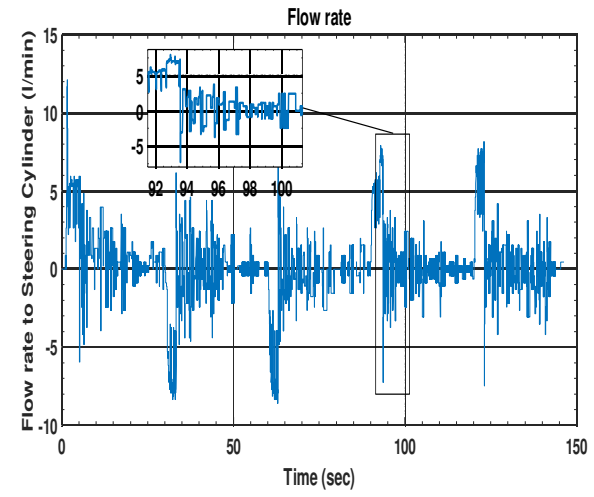

Fig. 8. Measured flow rate

\section{Conclusions}

The main result of the presented work is the synthesized $H_{\infty}$ controller forelectrohydraulic steering systems for the steering cylinder trajectory tracking. The controller design is based on the experimentally identified SIMO linear mathematical model. The experimental studies are performed on a laboratory test rig based on the 32-bit microcontroller. The advantages of the new controller are precise path tracking control and providing a variable steering ratio between the steering wheel and the steering cylinder.

More precisely, the controller provides transient response with the settling time of $10 \mathrm{~s}$ without overshoot, rise time of $5 \mathrm{~s}$ and ensures the control system performance in both directions of movement. The experimental results obtained show the workability and control performance of the designed system.

\section{Acknowledgements}

This work was supported by the Bulgarian Ministry of Education and Science under the National Research Programme "Low carbon energy for transport and live", approved by DCM \# 577/17.08.2018.

\section{References}

[1] Más F., Zhang Q. etc. Mechatronics and Intelligent Systems for Off-road Vehicles. SpringerVerlag London Limited, 2010.

[2] Ljung L. System Identification: Theory for the User. Second edition. Prentice-Hall Inc., Englewood Cliffs, NJ, 1999.

[3] Zhou K., Doyle J. etc. Robust and Optimal Control. Prentice Hall International Inc., Upper Saddle River, NJ, 1996.

[4] Petkov P., Slavov Ts., Kralev J. Design of Embedded Robust Control Systems using MATLAB®/Simulink®. IET Control, 2019.

[5] Milić V., Šitum Ž., Essert M. Robust Hœ position control synthesis of an electro-hydraulic servo system. ISA Transactions, vol. 49, 2010, pp. 535-542.

[6] Zhao W., Li Y., Wang C., Zhao T., Gu X. Ho control of novel active steering integrated with electric power steering function. Journal of Central South University, vol. 20, 2013, pp. 21512157.

[7] Jin Z., Zhang L., Zhang J., Khajepour A. Stability and optimized Ho control of tripped and untripped vehicle rollover. Vehicle System Dynamics, vol. 54, 2016, pp. 1405-1427.

[8] Dannöhl C., Müller S., Ulbrich H. Hœ-control of a rack-assisted electric power steering system. International Journal of Vehicle Mechanics and Mobility, vol. 50, 2012, pp. 527-544.

[9] Angelov Il., Mitov Al. Test Bench for Experimental Research and Identification of Electrohydraulic Steering Units. International Fluid Power Conference 10thIFK'2016, March, 2016, Dresden, Germany.

[10] Mitov Al., Kralev J., Slavov Ts., Angelov Il. SIMO System Identification of Transfer Function Model for Electrohydraulic Power Steering. 16thInternational Conference on Electrical Machines, Drives and Power Systems (ELMA), June, 2019, Bulgaria, pp. 130-135. 